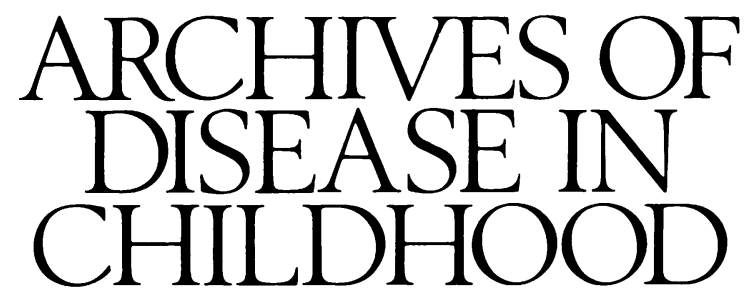

The Fournal of the British Paediatric Association

\title{
Annotations
}

\section{Treatment of the short, sexually immature adolescent boy}

Although constitutional delay of growth and puberty (CDGP) can be considered a normal variant, it remains the most common reason for boys presenting to an endocrine clinic. Many are referred because measurements by school doctors or general practitioners show them to be 'falling through' or 'falling away from' the height centiles or because of parents' concerns. Many boys will not perceive their short stature or lack of sexual development as a significant problem. Others may be distressed by lack of stature, sexual development or both. Pathology may need excluding but psychological stresses are not in any way dependent on its presence. ${ }^{1}$

In some, explanation may be all that is required. This must be based on knowledge of the natural history of pubertal development and growth, and an empathy with the psychological pressures that being 'different' in adolescence in terms of size and sexual development can bring. In others, emotional and psychological support will be necessary. However, it may be appropriate to attempt to bring forward the timing of the pubertal growth spurt and development of secondary characteristics by hormone treatment, not as an end in itself but to provide improved emotional well being. If hormonal intervention is necessary, in the present state of knowledge what advice can be given? Which treatments are effective? By what criteria? What are their disadvantages or dangers? Which are most acceptable to adolescents themselves? When should they be used?

The effects of growth promoting and puberty promoting interventions on short term growth and pubertal progression are becoming clearer and the paper by Albanese et al provides a useful practical pointer to the management of boys with CDGP. ${ }^{2}$ However, some questions and uncertainties remain. The recruitment criteria were very broad in terms of age and pubertal stage. Some would have been only just pubertal whereas others were well advanced with their growth spurt not far away even if untreated. The effects of oxandrolone vary with age and stage of puberty. Could oxandrolone have been as effective as testosterone in stimulating virilisation because a number of the subjects were maturing spontaneously as rapidly as those on testosterone in any case? No formal psychological assessment was made before recruitment and no psychological outcomes were measured. Would some of the more mature boys have been better treated with explanation and reassurance? The follow up period was very short and the effects of anabolic steroids on bone maturation, which may continue after treatment stops, could not be identified over such a time period.

This and a number of other short term studies of androgen, anabolic steroid, or growth hormone treatments have reported outcomes in sometimes diverse groups of patients, without long term follow up (off treatment or to final height), without psychological assessments and leave some important questions unanswered. Carefully controlled long term prospective trials to determine the criteria for considering treatment, the optimum regimen for growth stimulation and pubertal progression, and psychological outcomes in treated and untreated adolescents are necessary, and in progress.

Boys with CDGP, unless against a background of familial short stature, will reach a final height within the normal range. Increased adult height (even if achievable) may not be the most important anthropometric outcome in CDGP. Nevertheless, many may not reach their genetic height potential ${ }^{3}$ and in this study there was retrospective evidence of significant psychological distress in adolescence and a strong desire, in those questioned, to have received treatment to advance their growth spurt.

There is no substitute for long term follow up. Height prediction is fraught with difficulties particularly at around the time of puberty and is least accurate (and therefore least useful) in individuals showing marked deviations from the mean in terms of height, height velocity, and skeletal maturation. It is dependent on bone age estimation, which has an inherent assessment error that is greater between observers (often the case in multicentre growth trials) than when a single observer blinded to the clinical situation is responsible.

There could be other than psychological consequences of CDGP. The timing of puberty may be an important determinant of adult bone mineral density and those with CDGP could be at greater risk of osteoporosis in later adulthood. ${ }^{4}$

The full effects of anabolic steroids on bone maturation ${ }^{5}$ may not be evident for over six months after treatment is discontinued and skeletal maturation may continue to advance for up to a year. Thus when interpreting the 
effects of courses of anabolic steroids on growth, and in particular on predicted height, follow up data in the period after treatment are required. This is not reported in all studies (for example, Albanese et $\mathrm{al}^{2}$ ). Anabolic steroids may have a greater effect on skeletal maturation in younger children than in those whose bone age is approaching that of puberty. This may reflect a difference in sensitivity of the epiphyseal cartilage at an age when it is not normally subjected to androgens, or a greater resistance of more mature epiphyseal cartilage to exogenous influence since it is already under the influence of endogenous gonadal steroids. However, this may be a dose related phenomenon and effects on skeletal maturation (even when studied long term) need to be interpreted with caution and related to age, pubertal stage, dose and time on treatment.

Oxandrolone thus seems to be an excellent growth promoter in late prepubertal boys with significant growth and maturational delay, ${ }^{26}$ but there have not been ideally controlled studies in homogeneous groups of patients with sufficiently long follow up to be confident about its effects on sexual maturation in late prepuberty (as opposed to early to mid puberty) or on final height. Its effects on sexual maturation may be age and pubertal stage dependent - results obtained in a given age group at a particular pubertal stage cannot automatically be applied to other situations.

When oxandrolone is used, it should be in the minimum practical dose $\left(1 \cdot 25-2 \cdot 5 \mathrm{mg}\right.$ orally daily) ${ }^{5}$ Its availability long term is in doubt (partly because of its potential for, and documented, abuse by body builders and athletes) and is an unlicensed treatment currently available only on a named patient basis.

The commonly used injectable form of testosterone in the UK has been a mixture of long acting testosterone esters (Sustanon). There are surprisingly few detailed studies on its use in boys with puberty delay. Even with low dosage, supraphysiological levels of testosterone are unavoidable in the first 48-72 hours after injection, followed by gradual waning of effect over the next 3-4 weeks. In spontaneous pubertal development, more or less constant testosterone concentrations are found physiologically only when puberty is relatively far advanced. This pattern of testosterone exposure, with the depot released over weeks, is therefore very unphysiological compared with early male puberty, where there is a rise in testosterone in the morning (due to increasing nocturnal luteinising hormone pulsatility) followed by a fall during the day. ${ }^{89}$ These preparations must be given by repeated intramuscular injections, which are painful and disliked by many adolescents.

Testosterone undecanoate (17 $\beta$-hydroxy-4-androsten3 -one $17 \beta$-undecanoate) is an oral testosterone preparation in which testosterone has been esterified with undecanoic acid. This enhances the lipophilic character of the steroid, and thus it can be incorporated into chylomicrons in the intestinal wall. It is then protected from metabolism by intestinal wall enzymes and transported via lymphatics to the peripheral circulation before passing to the liver. It is most effective when administered as a solution in arachis oil, which itself stimulates the production of chylomicrons.

There have been past concerns about high levels of plasma androgens, especially dihydrotestosterone, after testosterone undecanoate administration ${ }^{10}$ and that $40 \mathrm{mg}$ daily might be too high an initial replacement dose. Recent detailed pharmacokinetic studies ${ }^{11} 12$ have evaluated doses of $40 \mathrm{mg}$ and of $20 \mathrm{mg}$ taken once daily after breakfast in prepubertal boys. Testosterone undecanoate absorption has been shown to match appropriately the diurnal pattern of early pubertal testosterone secretion. However, on the
$40 \mathrm{mg}$ regimen, peak concentrations appeared high (25-40 times basal value). Total testosterone concentrations were lower after three months and six months of treatment, with a parallel fall in sex hormone binding globulin. Concentrations of free testosterone remained constant during the treatment period, and were in an acceptable range (comparable with those found at Tanner stage 3 to 4 spontaneous puberty). High concentrations of dihydrotestosterone relative to testosterone were again found. ${ }^{11}$

It was felt ${ }^{11}$ that oral testosterone undecanoate would be a suitable method of puberty induction in boys but that a low dose ( $20 \mathrm{mg}$ daily) would be preferable. This was demonstrated to be the case in a double blind placebo controlled study using one (especially formulated) $20 \mathrm{mg}$ capsule daily. ${ }^{12}$ In this study, the boys taking testosterone undecanoate grew significantly faster than the placebo group without significantly faster bone age maturation and no differences were found between the groups in terms of growth hormone or gonadotrophin secretion or early morning testosterone concentrations.

Testosterone undecanoate is currently available only as $40 \mathrm{mg}$ capsules and is likely to be most physiological and effective at growth promotion (advancing the timing of the male pubertal growth spurt) and physiological sexual maturation when given as $40 \mathrm{mg}$ every other day. ${ }^{11} 12$ Used in a dose of $40 \mathrm{mg}$ daily ${ }^{11}$ it also seems effective but this regimen may be more appropriate for those boys already advancing in early puberty but in whom the growth spurt may still be up to two years distant.

It is inadvisable to give oral testosterone preparations for long periods because $17 \alpha$-alkyl testosterone derivatives can cause dose related (but reversible) cholestatic jaundice, but this is unnecessary in boys with CDGP or familial short stature in any case. In the pharmacokinetic studies liver function tests remained normal and no jaundice was seen. ${ }^{11} 12$ It is occasionally associated with nausea, although usually this passes off within a few days.

Thus boys presenting with delayed puberty can be treated effectively and cheaply with oral testosterone and, in the present state of knowledge, oral testosterone undecanoate ( $40 \mathrm{mg}$ alternate days or daily after breakfast) is probably the treatment of choice in comparison with anabolic steroids or injectable testosterone preparations. Certainly boys over 14 years of age, in whom pathology has been reasonably excluded and who have impaired self image and social withdrawal not responding to reassurance, should be considered for treatment and oral testosterone undecanoate appears safe and is generally effective in this context.

A role for recombinant human growth hormone treatment in boys with familial short stature and CDGP has been considered and is the subject of other current studies. The potential use of growth hormone in these contexts could usefully be explored further in studies concentrating on short term treatment regimens. There is currently a lack of such studies specifically designed to look at both medium term growth effects and final height in terms of, for example, optimal age for starting, dosage regimens, incremental doses, intermittent courses, 'catch-down' growth, and psychological outcomes. Such studies could provide data that would allow treatment to be optimised and, perhaps, cost effective. ${ }^{13}$

Much remains to be done in evaluating scientifically, in growth, pubertal and psychological terms, the most appropriate treatment interventions in different groups of 'short normal' children (with CDGP or familial short stature) at different ages. In such studies, which are in progress, assessing feelings is as important as assessing height, testicular volumes, bone mineral density, or 
hormone concentrations. Meanwhile, these aspects should be an important part of clinical decision making regarding the need for treatment. Effective interventions with hormone treatments, as well as psychological support, are now available when indicated and should not be denied when appropriate.

CHRISTOPHER J H KELNAR

Department of Child Life and Health,

17 Hatton Place,

Edinburgh EH9 $1 U W$

1 Kelnar CJH. Pride and prejudice - stature in perspective. Acta Paediatrica Scandinavica (Suppl) 1990; 370: 5-15.

2 Albanese A, Kewley GD, Long A, Pearl KN, Robins DG, Stanhope R. Oral therapy for constitutional delay of growth and puberty in boys: a therapy for constitutional delay of growth and puberty in boys: a
randomised trial of an anabolic steroid or testosterone undecanoate. Arch Dis Child 1994; 71: 315-7.

3 Crowne EC, Shalet SM, Wallace WHB, Eminson DM, Price DA. Final height in boys with untreated constitutional delay in growth and puberty. Arch Dis Child 1990; 65: 1109-12.

4 Finkelstein JS, Neer RM, Biller BMK, Crawford JD, Klibanski A. Osteopenia in men with a history of delayed puberty. N Engl f Med 1992; 326: $600-4$.

5 Sobel EH. Anabolic steroids. In: Astwood EV, Cassidy CE, eds. Clinical endocrinology II. New York: Grune and Stratton, 1968: 789-97.
6 Stanhope R, Brook CGD. Oxandrolone in low dose for constitutional delay of growth and puberty in boys. Arch Dis Child 1985; 60: 379-81.

7 Stanhope R, Buchanan CR, Fenn GC, Preece MA. Double-blind placebo controlled trial of low dose oxandrolone in treatment of boys with constitutional delay of growth and puberty. Arch Dis Child 1988; 63: 501-5.

8 Wu FCW, Butler GE, Kelnar CJH, Stirling HF, Huhtaniemi I. Patterns of pulsatile luteinizing hormone and follicle stimulating hormone secretion in prepubertal (midchildhood) boys and girls and patients with idiopathic in prepubertal (midchildhood) boys and girls and patients with idiopathic hypogonadotrophic hypogonadism (Kallmann's syndrome): a study using an ultra-sensitive time-resolved im
Endocrinol Metab 1991; 72: 1229-37.

9 Wu FCW, Butler GE, Kelnar CJH, Sellar RE. Patterns of pulsatile LH secretion before and during the onset of puberty in boys - a study using an immunoradiometric assay. 7 Clin Endocrinol Metab 1990; 70: 629-37.

10 Geere G, Jones J, Atherden SM, Grant DB. Plasma androgens after a single oral dose of testosterone undecanoate. Arch Dis Child 1980; 55: 218-20.

11 Butler GE, Sellar RE, Walker RF, Kelnar CJH, Wu FCW. Oral testosterone undecanoate in the management of delayed puberty in boys: pharmacokiundecanoate in the management of delayed puberty in boys: pharmacokinetics and effects on

12 Brown DC. Changes in hormones, growth and energy metabolism at the onset of male human puberty [MD thesis]. Belfast: Queen's University of Belfast, 1993.

13 Kelnar CJH, Tanaka T. Is there a place for short bursts of GH treatment in short children without significant GH deficiency? Acta Paediatrica 1994 (in press). (Summary of workshop at the 17th International Symposium on Growth and Growth Disorders, Barcelona April 15-16th 1994.) 